\title{
The autophagy process
}

\section{Sushil Devkota}

A day in the life of a cell,

There are so many stories to tell.

Ribosomes are cooking proteins, It smells delicious in cells kitchen; The beauty of the genetic code, Is inside the Nucleus laid hidden.

Kinases are busy kissing proteins, They leave the lip mark of phosphate on them; Phosphatases are running here and there with jealousy,

Removing phosphate is their game.

Of all these stories inside a cell,

Those are required to keep the cell neat;

This is the story of autophagy,

The process by which cells 'self-eat'.

When there is dark everywhere,

And not enough food on the table;

When cells feel unstable,

And bacteria, viruses, and oncogenes prevail.

A glimmer of hope emanates in the cytosol, Wearing the double membranous veil;

'So do not fear' Autophagy roars,

'I declare war against adversities and will prevail'.

Collecting intelligence cues from diverse signaling, Autophagy breaks shackles of mTOR;

ULKs and Class III PI3K complex next join the war, The first battalion is formed at the phagophore.

Mimicking the Ubiquitin system,

ATG5-ATG12-ATG16 then join the noble cause;

This war is about 'to be or not to be',

There is no excuse for rest or a pause.

Final reinforcement of LC3,

Ultimately decorates the lipid bilayer;

The membrane is going to form a circle,

Engulfed materials will soon disappear.
Nevertheless, like in any war,

Autophagy complains-'Rumors were spread against me';

'Autophagy is brutal'-it was told,

Engulfs and kills targets indiscriminately'.

Alas, they did not know,

Autophagy can be very selective;

Autophagy receptors p62 or NBR1,

Perform the selective autophagy trick.

Mitophagy for mitochondria, lipophagy for lipids, And pexophagy for the peroxisomes;

There are many secrets of autophagy hidden,

Those are yet to be known.

Autophagosome dumps the cargo on the lysosome, An organelle full of protease knife;

All the contents are then chopped to be recycled,

Executing the beautiful 'art-of-life'.

This is the story of Autophagy,

Keeping the cell clean its ultimate motive;

Autophagosome ends its life in the lysosome,

But the cell continues to live.

Sushil Devkota: Section of Cell and Developmental Biology, University of California, San Diego, La Jolla, CA, USA

Correspondence to: Sushil Devkota, email sdevkota@ucsd. edu

Keywords: autophagy, lysosome, molecular-signaling, protein-degradation

Received: February 22, 2017

Published: March 07, 2017 